\title{
Current perspectives on novel systemic therapeutic agents beyond immune checkpoint inhibition in metastatic urothelial carcinoma
}

\author{
Hoon Choi, Jae Young Park, Jae Hyun Bae, Bum Sik Tae \\ Department of Urology, Korea University Medical Center, Korea University School of Medicine, Ansan 15355, Korea \\ Contributions: (I) Conception and design: BS Tae; (II) Administrative support: JH Bae, BS Tae; (III) Provision of study materials or patients: All \\ authors; (IV) Collection and assembly of data: H Choi; (V) Data analysis and interpretation: H Choi; (VI) Manuscript writing: All authors; (VII) \\ Final approval of manuscript: All authors. \\ Correspondence to: Bum Sik Tae. Department of Urology, Korea University Ansan Hospital, Korea University College of Medicine, 123 Jeokgeum-ro, \\ Danwon-gu, Ansan 15355, Korea. Email: bumsik4ever@hanmail.net.
}

\begin{abstract}
Treatment strategies for metastatic urothelial carcinoma (mUC) have evolved dramatically over the last decade. The emergence of immunotherapeutic agents, especially immune checkpoint inhibitors, has been the most significant development. Immunotherapy increased the overall survival rate of patients with $\mathrm{mUC}$ and provided a durable response. The success of immune checkpoint inhibitors further led to the development of novel agents that regulate the immune system within the tumor microenvironment. However, despite some success with immune checkpoint inhibitors, researchers are still developing new agents, including small molecule tyrosine kinase inhibitors, mammalian target of rapamycin (mTOR) inhibitors, and novel fusion proteins, tailored for targets other than immune checkpoint inhibitors. Novel treatment strategies are being developed rapidly with ongoing pre-clinical trials. Here, we outline promising new therapies that are expected to improve survival rates for patients with mUC.
\end{abstract}

Keywords: Metastatic urothelial carcinoma (mUC); chemotherapy; novel

Submitted Mar 25, 2020. Accepted for publication Apr 23, 2020.

doi: $10.21037 /$ tcr-20-1262

View this article at: http://dx.doi.org/10.21037/tcr-20-1262

\section{Introduction}

In metastatic urothelial carcinoma (mUC), $15 \%$ of patients show long-term survival after cisplatin-based chemotherapy; in patients with recurrent disease, the response rate to secondary chemotherapy is typically $15-20 \%$, with a 3 -month progression free survival (1). Platinum-based chemotherapy has long been the treatment of choice for metastatic UC as a metastatic line treatment. Cisplatin based chemotherapy has been firmly established in the treatment of patients with metastatic UC for decades. The National Comprehensive Cancer Network (NCCN) guidelines recommend cisplatin-based chemotherapy as standard-ofcare in perioperative (as neoadjuvant and adjuvant therapy) and first-line treatment (2). Concomitant chemotherapy of cisplatin with gemcitabine or methotrexate/vinblastine/ doxorubicin is recommended for patients with advanced surgical insufficiency or metastatic disease that can tolerate cisplatin (3).

However, recent advances in the development of novel agents, including immunotherapeutic agents, has significantly improved the survival outcomes of patients with metastatic UC. Single agent immune checkpoint inhibitors (ICIs), including anti-programmed cell death protein 1 (PD-1)/programmed death-ligand 1 (PD-L1) antibodies, are emerging as treatment options for patients who have undergone cytotoxic systemic chemotherapy. However, many patients do not respond to a single agent ICI. Therefore, developing additional treatment options with biological theoretical basis at mUC remains a priority for researchers. Novel treatment strategies are being 
developed rapidly and pre-clinical trials are ongoing. Here, we have summarized systemic treatment options for $\mathrm{mUC}$, with an emphasis on approved next treatment options and later stage investigations beyond the ICIs.

\section{Targeted therapy}

The advent of immunotherapy has drastically improved the treatment options for metastatic UC. However, durable responses are only observed in some patients, and response rates do not exceed $20 \%$ under first-line or post chemotherapy settings. Similarly, targeted therapies have not had a good overall response rate and have rarely succeeded in metastatic UC models. However, previous preclinical results have suggested the possibility that some anti-angiogenic factors and tyrosine kinase inhibitors (TKIs) may increase the effectiveness of immunotherapeutic agents in the tumor microenvironment (4). Therefore, many clinical trials are underway to evaluate the effectiveness of new agents in combination with immunotherapy in treating metastatic UC.

\section{Vascular endothelial growth factor receptor}

Previous studies have shown that elevated level of vascular endothelial growth factor receptor (VEGF) in the urinary tract and serum of patients with $\mathrm{UC}$ is associated with an increase in the number of aggressive tumors, with poor prognosis $(5,6)$. Currently, two phase II trials (NCT03133390 and NCT03272217) are underway to investigate effects of bevacizumab with atezolizumab on cisplatin-ineligible patients and previously untreated $\mathrm{mUC}$ (Table 1). The second agent that targets VEGF signaling is ramucirumab, a monoclonal antibody that targets VEGFR2. Ramucirumab has been studied in combination with pembrolizumab in a phase I multiple cohort study of patients with UC who recently underwent platinum-based systemic therapy. Patients treated with the combination therapy suffered acceptable treatment-related adverse events and showed objective antitumor activity (7). Notably, patients in the ramucirumab and docetaxel group had a mean progression free survival (mPFS) of 4.07 months, compared with 2.76 months in the phase III study on 530 patients. In addition, an objective response of $24.5 \%$ for patients assigned to the ramucirumab group and $14.0 \%$ (95\% CI: 9.4-18.6) for patients assigned to the placebo group was noted. These results are noteworthy; they represent the first novel agents demonstrating progression free survival (PFS) advantages over other chemotherapeutics in a post-platinum setting and presenting VEGF2 inhibition as a therapeutic pathway in metastatic UC (8). The results of the RANGE trial suggest that VEFG2 inhibition can have many potential benefits in the future. Another strategy for VEGF inhibition involves novel recombinant EphB4HSA fusion proteins. EphB4-HSA is being evaluated in combination with pembrolizumab in the treatment of naïve IV UC as a phase II study (NCT02717156).

In contrast, cabozantinib, a small molecule TKI that targets receptors such as RET, KIT, AXL, FLT3, MET, and VEGFR2, has recently been approved for metastatic RCC (mRCC) in secondary environment, after the METeOR test, and has been tested in various settings in various clinical trials (9). In phase I trials of patients who did not receive immunotherapy, cabozantinib combination with CPI showed mPFS in 12.8 months (95\% CI: $1.8-\mathrm{N} / \mathrm{A}$ ) and $70.2 \%$ (95\% CI: 44.4-85.8\%) overall survival (OS) (10). Cabozantinib with different CPIs, including pembrolizumab and atezolizumab, is also being investigated (NCT03534804, NCT03170960).

\section{Human epidermal growth factor receptor (HER)}

HER activation is related with tumor cell growth, proliferation, and chemotherapy resistance. The HER family has been extensively studied and is known to be a major target in the treatment of gastrointestinal and mammary malignancies. UC has one of the highest rates of HER2 expression (11). However, in a study of HER1/HER2-positive status, confirmed by centralized immunohistochemistry, in 232 patients with metastatic bladder cancer who were treated with primary chemotherapy, no improvement of PFS was observed in a comparative study between lapatinib maintenance and placebo (12).

However, afatinib, a dual EGFR and HER2 inhibitor, was shown to demonstrate clinical activity in platinumrefractory patients with UC, with mutations in ERBB2 or ERBB3, detected by quantitative polymerase chain reaction or fluorescent in situ hybridization (13). The study showed that five of six patients with mutations in ERBB2, ERBB3, or both achieved the 3 -month primary endpoint when compared with the 15 patients without these mutations. The average time of progression or discontinuation of treatment was 6.6 months in patients with ERBB2/ ERBB3 mutations and 1.4 months in patients without these mutations. Promising results with afatinib, a pan- 


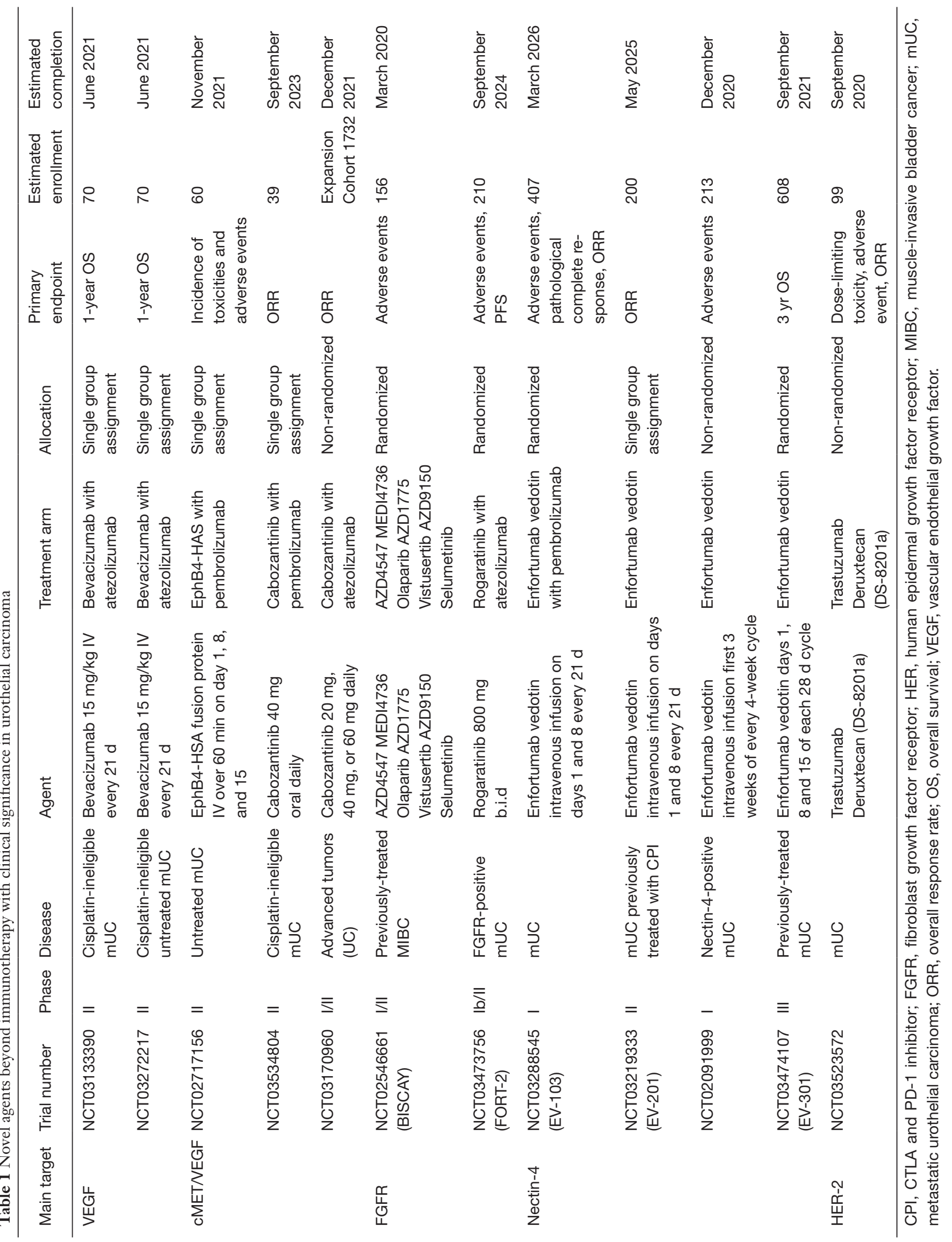


HER inhibitor for patients with mutations in HER, have led to larger studies, which have verified the results found in selected patients with metastatic UC. However, results from HER2 targeting treatment do not clearly show the clinical efficacy of unselected patients with UC. Therefore, a novel antibody-drug conjugate, trastuzumab deruxtecan, has been developed, and is being studied in combination with nivolumab in a multiple cohort phase I trial involving patients with UC (NCT03523572).

\section{Nectin}

Nectin-4 is a membrane-penetrating polypeptide involved in cell-adhesion and has been known for its role in tumor growth and blood vessel formation (14). Challita-Eid et al. used suppressive subtractive hybridization in pathological specimens of UC and showed high mRNA expression of nectin-4 (15). Further, they successfully produced enfortumab vedotin, a novel antibody-drug conjugate, consisting of a monoclonal antibody against nectin-4 bound to monomethyl auristatin $\mathrm{E}$, a potent cytotoxic microtubule inhibitor. In a phase I dose escalation study using enfortumab vedotin in 68 patients with metastatic UC, ORR was $41 \%$ and disease control rate was $72 \%$. Moreover, a high toxicity tolerance was observed, where only $9 \%$ of patients showed grade $3 / 4$ treatment-related adverse events (16). At the 2019 ASCO annual meeting, researchers presented a study where enfortumab vedotin induced a $44 \%$ response rate in patients with locally advanced or metastatic UC in the single-arm phase II EV-201 trial setting with a complete response of $12 \%$. The EV-201 trial enrolled patients with disease progression after treatment with platinum-based chemotherapy and/or checkpoint inhibitors. Mean overall survival (OS), mean PFS, and mean response duration (mDOR) were 11.7, 5.8, and 7.6 months, respectively, and most patients showed a well-tolerated adverse effect profile (17). Enfortumab vedotin is now in the spotlight as the first new therapeutic agent to demonstrate clinical benefits in patients progressing after CTLA and PD-1 inhibitor (CPI) treatment; phase III (EV-301 and EV-201) trials are currently underway.

\section{Fibroblast growth factor receptor}

The fibroblast growth factor (FGF) pathway is another well described tyrosine kinase (TK) signaling pathway implicated in tumor formation, with a high mutation rate in UC (18). Previous research has shown that mutations in fibroblast growth factor receptor (FGFR) are presented in nearly 15-20\% of metastatic UC (19). Erdafitinib (BAY1163877) and rogaratinib (FGFR1-4) are relatively selective and potent pan-FGFR inhibitors. As the first TKI approved for UC therapy, erdafitinib showed $32.3 \%$ ORR and $2.3 \%$ $\mathrm{CR}$ in a clinical trial involving 87 patients with advanced bladder cancer, with mutations in FGFR2 or FGFR3. BGJ-398 is also a pan-FGFR inhibitor studied in patients with metastatic UC. In a phase I clinical trial involving previously-treated patients with metastatic UC, with mutations in FGFR3, BGJ-398 showed a disease control rate of $64.2 \%$ and genomic FGFR 3 mutations exhibiting an overall response rate of $25.4 \%$ (20).

These notable studies have led to other studies examining the effectiveness of anti-FGF therapy in metastatic UC. The most notable of these was the BISCAY I phase study, which was conducted with the powerful and selective FGFR inhibitor AZD4547 in patients with FGFR3 mutations (NCT02546661). In addition, vofatamab (BZarrabi 701) is another new FGFR3 inhibitor that is being studied in combination with pembrolizumab in the FIERCE-22 international phase I/II trial of metastatic UC (NCT03123055). The therapeutic potential of the novel pan-FGFR inhibitor, rogaratinib (BAY1163877), is being tested in a variety of solid tumors, including metastatic $\mathrm{UC}$, in the FORT-2 test as a combination therapy with atezolizumab (NCT03473756).

\section{Diversification of chemotherapy}

Despite the remarkable development of ICIs, the overall response rate has not been high, as somatic mutation in DNA damage repair related genes is associated with neoadjuvant chemotherapy and PD1/PD-L1 blockade. This phenomenon has been demonstrated in clinical trials where ERCC2 mutations have been associated with survival in individual cohorts of patients receiving neoadjuvant chemotherapy for muscle-invasive bladder cancer (MIBC) from previous clinical trials (21). In addition, in a study of exon capture sequencing in 100 patients, mutations in DDR were associated with PFS and OS, and deletion of DNA damage repair genes, including RB1, ATM, and FANCC, was associated with the response to neoadjuvant chemotherapy (22). In a previous study, 22 to half of the patients with urothelial cell carcinoma had pathogenic mutations in DNA damage repair genes, such as CHEK2, BRCA1, BRCA2, ATM, BRIP1, and NBN. Ochoa et al. have suggested that expression of non-coding microRNAs 
can contribute to the control of gene expression patterns and identify basal or luminal muscle-invasive UC subtypes, which can be used as biomarkers for tumors, leading to targeted therapies (23). Molecular subtyping can predict the response of chemotherapy to patients who do not respond to ICI.

\section{Future considerations}

Innovative advances in ICI formulations in the treatment of metastatic UC have been a source of hope for many patients. Although beyond the focus of this review, in the MIBC setting, a number of phase II or III studies that incorporate ICI are underway. It is also approaching variously according to various spectrum of diseases, and ICI preparation is expected to play a bigger role in the future. In addition, using a single agent, like pembrolizumab or atezolizumab, appeared to be a feasible adjuvant therapy with a promising complete response rate. There are several I-III non-MIBC studies that examine the effects of systemic ICI, with or without intravenous therapy, in patients who do not respond to intravesical BCG beyond the metastatic setting, in various applications during disease progression. The prospect of ICI treatment is promising. However, because not all single agent therapies are efficient, various studies are underway for patients who do not respond to ICI, which has led to the development of several novel agents.

Novel possible targets in the metastatic environment can be derived from a better understanding of tumor biology based on a broader UC spectrum. Recently, a comprehensive analysis of MIBC using the TCGA analysis platform has been made to detect potential therapeutics by detecting APOBEC mutations and exploring the association of chromatin-mutated gene mutations in the early stages of disease treatment (24). Ongoing research on new targets beyond ICI will help us understand tumor biology better. However, the development of novel agents, comparable to ICI products, is still pending. It is hoped that in the future, various novel agents will be developed in various settings to help patient survival.

\section{Acknowledgments}

Funding: This work was supported by the National Research Foundation of Korea (NRF) grant (No. 2019R1F1A1058422) funded by the Korean government (MSIT).

\section{Footnote}

Provenance and Peer Review: This article was commissioned by the Guest Editors (Dr. Ja Hyeon Ku, Dr. Hyeong Dong Yuk, and Dr. Hyung Suk Kim) for the series "Urothelial Carcinoma" published in Translational Cancer Research. The article was sent for external peer review organized by the Guest Editors and the editorial office.

Conflicts of Interest: All authors have completed the ICMJE uniform disclosure form (available at http:// dx.doi.org/10.21037/tcr-20-1262). The series "Urothelial Carcinoma" was commissioned by the editorial office without any funding or sponsorship. The authors have no other conflicts of interest to declare.

Ethical Statement: The authors are accountable for all aspects of the work in ensuring that questions related to the accuracy or integrity of any part of the work are appropriately investigated and resolved.

Open Access Statement: This is an Open Access article distributed in accordance with the Creative Commons Attribution-NonCommercial-NoDerivs 4.0 International License (CC BY-NC-ND 4.0), which permits the noncommercial replication and distribution of the article with the strict proviso that no changes or edits are made and the original work is properly cited (including links to both the formal publication through the relevant DOI and the license). See: https://creativecommons.org/licenses/by-nc-nd/4.0/.

\section{References}

1. Iyer G, Rosenberg JE. Novel therapies in urothelial carcinoma: a biomarker-driven approach. Ann Oncol 2018;29:2302-12.

2. Flaig TW, Spiess PE, Agarwal N, et al. NCCN guidelines insights: Bladder cancer, version 5.2018. J Natl Compr Canc Netw 2018;16:1041-53.

3. Bellmunt J, Orsola A, Leow JJ, et al. Bladder cancer: ESMO practice guidelines for diagnosis, treatment and follow-up. Ann Oncol 2014;25:iii40-8.

4. Motz GT, Coukos G. The parallel lives of angiogenesis and immunosuppression: cancer and other tales. Nat Rev Immunol 2011;11:702-11.

5. Sankhwar M, Sankhwar SN, Abhishek A, et al. Clinical significance of the VEGF level in urinary bladder carcinoma. Cancer Biomark 2015;15:349-55. 
6. Cumberbatch K, He T, Thorogood Z, et al. Emerging drugs for urothelial (bladder) cancer. Expert Opin Emerg Drugs 2017;22:149-64.

7. Petrylak DP, Arkenau HT, Perez-Gracia JL, et al. A multicohort phase I study of ramucirumab (R) plus pembrolizumab $(\mathrm{P})$ : Interim safety and clinical activity in patients with urothelial carcinoma. J Clin Oncol 2017;35:349.

8. Petrylak DP, de Wit R, Chi KN, et al. Ramucirumab plus docetaxel versus placebo plus docetaxel in patients with locally advanced or metastatic urothelial carcinoma after platinum-based therapy (RANGE): a randomised, doubleblind, phase 3 trial. Lancet 2017;390:2266-77.

9. Choueiri TK, Escudier B, Powles T, et al. Cabozantinib versus everolimus in advanced renal cell carcinoma (METEOR): final results from a randomised, open-label, phase 3 trial. Lancet Oncol 2016;17:917-27.

10. Nadal RM, Mortazavi A, Stein M, et al. Results of phase I plus expansion cohorts of cabozantinib (Cabo) plus nivolumab (Nivo) and CaboNivo plus ipilimumab (Ipi) in patients (pts) with metastatic urothelial carcinoma (mUC) and other genitourinary (GU) malignancies. J Clin Oncol 2018;36:515.

11. Yan M, Schwaederle M, Arguello D, et al. HER2 expression status in diverse cancers: review of results from 37,992 patients. Cancer Metastasis Rev 2015;34:157-64.

12. Powles T, Huddart RA, Elliott T, et al. A phase II/III, double-blind, randomized trial comparing maintenance lapatinib versus placebo after first line chemotherapy in HER1/2 positive metastatic bladder cancer patients. J Clin Oncol 2015;33:4505.

13. Choudhury NJ, Campanile A, Antic T, et al. Stadler afatinib activity in platinum-refractory metastatic urothelial carcinoma in patients with ERBB alterations. J Clin Oncol 2016;34:2165-71.

14. Nishiwada S, Sho M, Yasuda S, et al. Nectin-4 expression contributes to tumor proliferation, angiogenesis and patient prognosis in human pancreatic cancer. J Exp Clin Cancer Res 2015;34:30.

Cite this article as: Choi H, Park JY, Bae JH, Tae BS. Current perspectives on novel systemic therapeutic agents beyond immune checkpoint inhibition in metastatic urothelial carcinoma. Transl Cancer Res 2020;9(10):6583-6588. doi: $10.21037 /$ tcr-20-1262
15. Challita-Eid PM, Satpayev D, Yang P, et al. Enfortumab vedotin antibody-drug conjugate targeting nectin-4 is a highly potent therapeutic agent in multiple preclinical cancer models. Cancer Res 2016;76:3003-13.

16. Rosenberg J, Sridhar SS, Zhang J, et al. EV-101: A phase I Study of single-agent enfortumab vedotin in patients with nectin-4-positive solid tumors, including metastatic urothelial carcinoma. J Clin Oncol 2020;38:1041-9.

17. Petrylak DP, Balar AV, O'Donnell PH, et al. EV-201: Results of enfortumab vedotin monotherapy for locally advanced or metastatic urothelial cancer previously treated with platinum and immune checkpoint inhibitors. J Clin Oncol 2019;37:4505.

18. Godwin JL, Hoffman-Censits J, Plimack E. Recent developments in the treatment of advanced bladder cancer. Urol Oncol 2018;36:109-14.

19. Li Q, Bagrodia A, Cha EK, Coleman JA. Prognostic genetic signatures in upper tract urothelial carcinoma. Curr Urol Rep 2016;17:12.

20. Pal SK, Rosenberg JE, Hoffman-Censits JH, et al. Efficacy of BGJ398, a fibroblast growth factor receptor 1-3 inhibitor, in patients with previously treated advanced urothelial carcinoma with FGFR3 alterations. Cancer Discov 2018;8:812-21.

21. Liu D, Plimack ER, Hoffman-Censits J, et al. Clinical validation of chemotherapy response biomarker ERCC2 in muscle-invasive urothelial bladder carcinoma. JAMA Oncol 2016;2:1094-6.

22. Plimack ER, Dunbrack RL, Brennan TA, et al. Defects in DNA repair genes predict response to neoadjuvant cisplatin-based chemotherapy in muscle-invasive bladder cancer. Eur Urol 2015;68:959-67.

23. Ochoa AE, Choi W, Su X, et al. Specific micro-RNA expression patterns distinguish the basal and luminal subtypes of muscle-invasive bladder cancer. Oncotarget 2016;7:80164-74.

24. Robertson AG, Kim J, Al-Ahmadie H, et al. Comprehensive molecular characterization of muscleinvasive bladder cancer. Cell 2018;174:1033. 